\section{Printing gray-scale graphics using mean dot density}

\author{
M. G. HARRIS \\ University of Birmingham, Birmingham, England
}

Printing gray-scale graphics using mean dot density rather than standard characters allows more continuous representation of the luminance dimension with little or no loss of spatial resolution.

Recently, White, Brussell, Williams, and Rog (1984) reemphasized the usefulness of plotting three-dimensional (3-D) data as 2-D maps in which the $\mathrm{z}$ axis is represented by luminance. The main purpose of their article was to provide a set of standard characters suitable for the production of such maps on dot-matrix printers. Most modern dot-matrix and daisy-wheel printers have a graphics mode that allows the printing of individual dots with high spatial resolution. This paper outlines a simple way of using this facility to produce maps in which the luminance dimension can be represented more or less continuously, rather than in the relatively few discrete steps practicable with character-based graphics.

The principle is very simple and uses local dot density to represent luminance. One simply arranges that the probability of a dot in each location be inversely proportional to the required luminance. In practice, a random number in the same range as the luminance data is generated at each coordinate, and a dot is plotted only if this number is greater than the luminance at that coordinate. If one is concerned with brightness rather than luminance, an appropriate transform (e.g., logarithmic) can be applied to the random numbers or the luminance data before they are compared.

Since dot-matrix printers often produce their output in a series of horizontal scans in which several vertical dots are printed simultaneously, it may be necessary to build the map in this format. The accompanying PASCAL segment (see Appendix) prints seven horizontal lines at a time by building each scan as a sequence of 7-bit patterns in which Bit 0 refers to the topmost dot. This segment assumes that the luminance data are stored in a 2-D array with the same spatial resolution as the printed map. If necessary, the resolutions of the luminance array and printed map can easily be matched by interpolation based on, for instance, the sinc function (Bracewell, 1978).

Figure 1 illustrates the use of this method to produce

The author's mailing address is: Department of Psychology, University of Birmingham, P.O. Box 363, Birmingham B15 2TT, England.

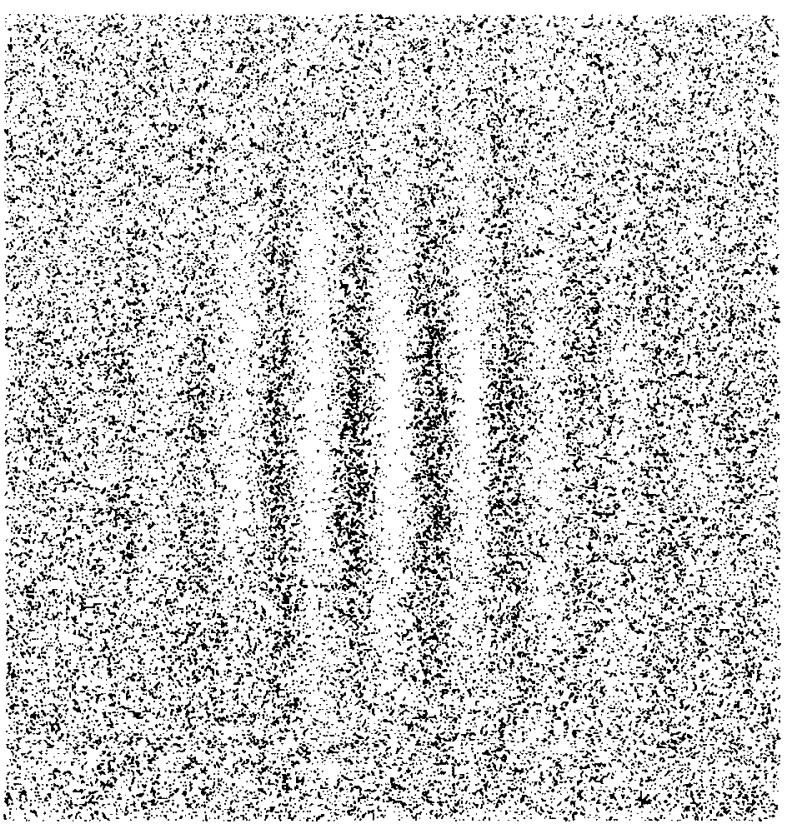

Figure 1. Luminance map of a 2-D Gabor function. The local probability of a dot, $p(x, y)=\bar{p}[1-G(x, y, \sigma) \cdot \cos (f x)]$, where $\bar{p}$ is mean probability, $G$ is a $2-D$ Gaussian function, and $f$ is $\mathbf{2} \pi$ /period. See text for parameters.

a luminance map of a Gabor function. This elementary stimulus, much used in visual psychophysics, consists of a vertical cosine grating with contrast weighted by a radially symmetric Gaussian. In this example, the cosine grating has a spatial period one-tenth the map width, and the Gaussian has a standard deviation one-fifth the map width. The original was produced on a Qume daisy-wheel printer using a resolution of 60 horizontal $\times 48$ vertical dots per inch. It measured $10 \times 10 \mathrm{in}$. and was designed to be viewed from a distance of about 10 times the map width. The mean dot probability is .2 , giving a mean density in the original of 576 dots/in. ${ }^{2}$ and a nominal peak contrast of 0.25 . Incidentally, this type of printed output is particularly suitable for photocopying, since it is relatively unaffected by the high-pass filtering of the photocopying process.

\section{REFERENCES}

BRACEWELL, R. N. (1978). The Fourier Transform and its applications (2nd ed.). Tokyo:McGraw-Hill Kogakusha.

White, C. W., Brussel, E. M., Williams, T. T., Rog, S. J. (1984).

Gray-scale graphics using dot-matrix printers. Behavior Research Methods, Instruments, \& Computers, 16, 273-276. 


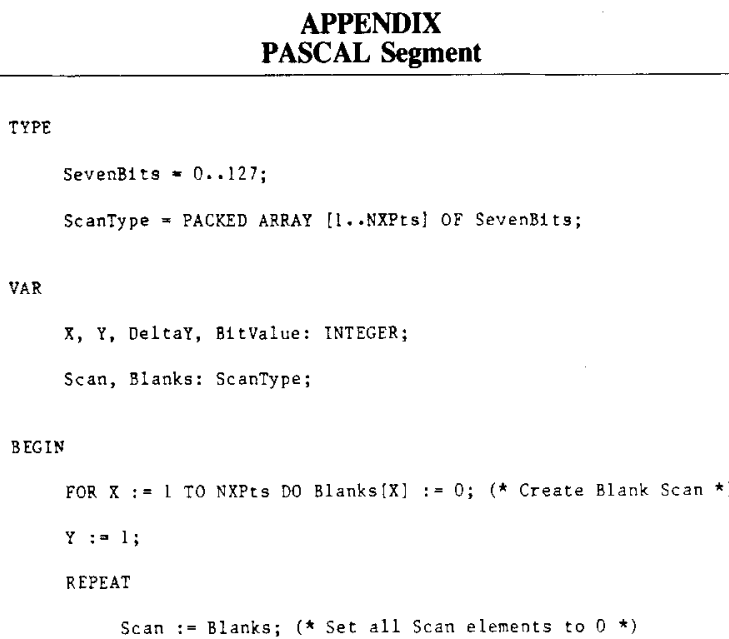

APPENDIX

PASCAL Segment

TYPE

SevenB1ts $=0 . .127$

Scantype = PACKED ARRAY [?,.NXPts] OE SevenB1tg;

VAR

$X, Y$, DeltaY, B1tValue: INTEGER

Scan, Blanks: ScanType;

BEGIN

FOR $X:=1$ TO NXPts DO Blanks $[X]:=0 ;$ (* Create Blank Scan *)

Y : : 1 ;

REPEAT

Scan : = Blanks; (* Set all Scan elements to 0 *)

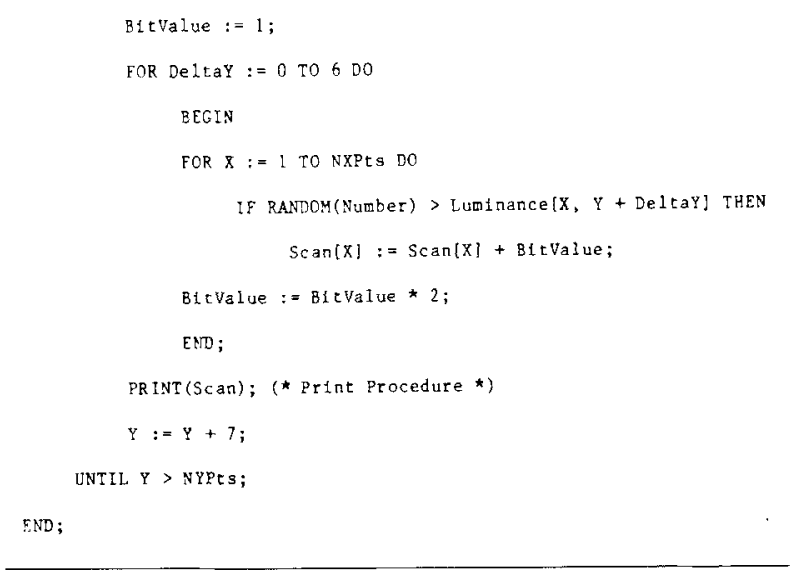

(Manuscript accepted for publication December 14, 1984.)

\section{Announcement}

\section{Artificial Intelligence Consortium Founded}

Eight universities have entered into a 5-year, $\$ 8,236,000$ contract with the U.S. Air Force's Rome Air Development Center (RADC) to study artificial intelligence - the application of computer systems to problems that could previously be solved only by human intelligence.

"The project is aimed at establishing a major research force in artificial intelligence in the Northeast," said Fred Diamond, chief scientist at the RADC at Griffiss Air Force Base in Rome, NY.

Members of the new Artificial Intelligence Consortium (AIC) are: Syracuse University, University of Rochester, Rochester Institute of Technology, State University of New York at Buffalo, Rensselaer Polytechnic Institute, Clarkson University, Colgate University, and University of Massachusetts. The Air Force Institute of Technology at Wright-Patterson Air Force Base in Ohio will participate in the program on a nonfunded basis. This unique cooperative effort is cosponsored by the Rome Air Development Center and the Air Force Office of Scientific Research, both organizations of the Air Force Systems Command.

Preparation of the funding proposal was organized and directed by Virgil Eveleigh, professor of electrical and computer engineering at Syracuse University, with major input by Syracuse University professors P. Bruce Berra, also in electrical and computer engineering, and Kenneth Bowen of the School of Computer and Information Science. Syracuse University will be the prime contractor with the Air Force and will negotiate subcontracting agreements with the other member institutions.

Although Syracuse University is the focal point for the AI Consortium, each member institution will contribute researchon an equal basis-in its respective area of expertise. Roles may change or expand as the project matures.

Initial research interests of the member institutions are:

-Syracuse University-logic programming and the development of special-purpose computer hardware for typical AI applications;

-University of Rochester-problem solving, and temporal relationships and reasonings (which involves ordering the actions of devices monitored by a computer);

- Rochester Institute of Technology-speech-understanding systems, including microphone speech processing;

-SUNY Buffalo-versatile expert maintenance systems;

-Rennselaer Polytechnic Institute-image-understanding systems, aerial and satellite imagery (both electronic and photographic);

-Clarkson University-distributed problem solving;

- Colgate University-natural language processing and plan recognition research;

-University of Massachusetts-intelligent user interfaces, natural language generation and understanding, and distributed artificial intelligence (which involves the coordination of expert systems).

The Artificial Intelligence Consortium has four main goals: to improve the Air Force's technical AI abilities; to improve the training of Air Force technicians and scientists and expand the general base of AI expertise; to stimulate business involvement in AI research and development; and to establish a productive, cooperative relationship among institutions distinguished by their research in this field.

Scientists and engineers believe AI techniques will enable them to develop advanced computer systems that not only can process vast amounts of information, but also can interpret, predict, analyze, and explain such data.

For further information, contact: Anthony D'Angelo, Syracuse University, 105 Administration Building, Syracuse, NY 13210. 